\title{
Delay analysis of a HOL priority
}

\section{queue}

\author{
Joris Walraevens, Bart Steyaert, Marc Moeneclaey and Herwig Bruneel \\ Ghent University - UGent \\ Department of Telecommunications and Information Processing (TW07) \\ Sint-Pietersnieuwstraat 41, B-9000 Gent, Belgium.
}

Phone: +32-9-2648902

Fax: +32-9-2644295

E-mail: $\{j w, b s, m m, h b\} @ t e l i n . U G e n t . b e$

\begin{abstract}
Priority scheduling for packets is a hot topic, as interactive (voice,video) services are being integrated in existing data networks. In this paper, we consider a discrete-time queueing system with a non-preemptive (or Head-Of-the-Line) priority scheduling discipline and a general number of priority classes. Packets of variable length arrive in the queueing system. We derive expressions for the probability generating functions of the packet delays. From these functions, some performance measures (such as moments and approximate probabilities) are calculated. We illustrate the performance measures by means of some numerical examples and show how to apply the theoretical results to a queue that handles arriving multimedia traffic.
\end{abstract}




\section{Introduction}

In recent years, there has been much interest in incorporating multimedia applications in packetbased networks (e.g. IP networks). Different types of traffic need different QoS standards. For real-time interactive applications, it is important that mean delay and delay-jitter are bound, while for non real-time applications, the loss ratio is the restrictive quantity. In order to guarantee acceptable delay boundaries to delay-sensitive traffic (such as voice/video), several schedul-

ing schemes - for switches, routers, ...- have been proposed and analyzed, each with their own specific algorithmic and computational complexity.

Amongst these scheduling disciplines are some well-known strategies like weighted roundrobin (WRR), weighted fair queueing (WFQ) or generalized processor sharing (GPS), earliest deadline first (EDF), probabilistic priority (PP) and (strict) priority scheduling. In a queueing system with WRR (see e.g. Liu et al. (1997) and references therein), WFQ or GPS (see e.g. Parekh and Gallager (1994) and references), the server serves a number of queues by a weighted schedule. Delay sensitive traffic is assigned a higher weight, i.e., (in average) delay-sensitive traffic is earlier/longer served than delay-insensitive traffic. When the EDF scheduling is applied, deadlines are imposed on the packets that have to be served (based on their QoS constraints) and packets are transmitted in the order of their deadlines (see Liebeherr and Wrege (1999) and references therein). A PP scheduling discipline (see e.g. Tham et al. (2002)) serves a given number of priority queues in a probabilistic manner. Each priority queue is assigned a parameter $p_{i}$, which determines the probability that a packet from that priority queue is served next.

All these scheduling disciplines try to give some kind of priority to delay-sensitive traffic over delay-insensitive traffic. The most drastic in this respect is the strict priority scheduling. With this scheduling, packets are classified in a number of priority classes (based on their delay constraints). As long as packets of the highest priority class are present in the queueing system, this type of traffic is served. Packets of lower priority can only be transmitted when no higher priority traffic is present in the system. As already mentioned, this is the most drastic way 
to meet the QoS constraints of delay-sensitive traffic (and thus the scheduling with the most disadvantageous consequences on the delay-insensitive traffic), but also the easiest to implement.

In this paper, we focus on the effect of a non-preemptive or HOL (Head-Of-the-Line) priority scheduling discipline on the performance of a queue with multiple traffic classes. More delaysensitive traffic is assumed to have non-preemptive priority over traffic with more flexible delay constraints, i.e., when the server becomes idle, a packet of the most delay-sensitive traffic that is available is scheduled for service next. Newly arriving packets cannot interrupt the transmission of a packet that has already commenced, whatever their priority level. This is a situation that occurs in some of the service models that have been proposed for packet-based networks, such as the Differentiated Service model for IP networks (Xiao and Ni, 1999).

In the literature, there have been a number of contributions with respect to HOL priority scheduling. An overview of some basic HOL priority queueing models in continuous time can be found in Miller (1960), Kleinrock (1976) and Takagi (1991) and the references therein. Khamisy and Sidi (1992), Takine et al. (1994b), Laevens and Bruneel (1998) and Walraevens et al. (2003) have studied discrete-time HOL priority queues with deterministic service times equal to one slot. Khamisy and Sidi (1992) analyze the system contents of the different classes, for a queue fed by a two-state Markov modulated arrival process. In Takine et al. (1994b), the system content and the delay for Markov modulated high-priority arrivals and geometrically distributed low-priority arrivals are presented. Laevens and Bruneel (1998) analyze the system content and cell delay in the case of a multi-server queue. Walraevens et al. (2003) study the system content and packet delay, in the special case of an output queueing switch with Bernoulli arrivals. Furthermore, non-preemptive HOL priority queues have been considered by Rubin and Tsai (1989), Stanford (1991), Takahashi and Hashida (1991), Takine et al. (1994a), Sugahara et al. (1995), Takine (1996), Walraevens et al. (2000) and Walraevens et al. (2002). Rubin and Tsai (1989) study the mean waiting time, for a discrete-time queue fed by an i.i.d. arrival process. Stanford (1991) analyzes the inter-departure time distribution in a queue fed by a Poisson process in continuous time. Takahashi and Hashida (1991) study the packet delay of a priority queue with a general 
number of priority classes, using an analysis based on the calculation of the unfinished work and delay-cycles. Sugahara et al. (1995) present a non-preemptive queue in continuous time, with a Switched Poisson arrival process for the high-priority packets. Takine et al. (1994a) and Takine (1996) study discrete-time MAP/G/1 queues, using matrix-analytic techniques. Finally, Walraevens et al. (2000) and Walraevens et al. (2002) analyze the system content and packet delay respectively in a two-class discrete-time priority queue with general service times and i.i.d. numbers of per-slot arrivals.

In this paper, we analyze the packet delays of all priority classes in a discrete-time singleserver buffer with infinite buffer space for a non-preemptive HOL priority scheme and per-slot i.i.d. arrivals and a general number of classes. The transmission times of the packets are assumed to be generally distributed and class-dependent (which reflects the case where different classes represent different applications). Furthermore, the numbers of per-slot arrivals of different classes may be correlated. We will demonstrate that an analysis based on probability generating functions is extremely suitable for modeling this type of buffers with a priority scheduling discipline. From these probability generating functions, we can then easily calculate expressions for some interesting performance measures, such as the moments and tail probabilities of the packet delay of all traffic types. These closed-form expressions require virtually no computational effort at all, and are well-suited for evaluating the impact of the various system parameters on the overall performance. This makes it possible to study the effect of a HOL priority scheduling on the packet delay of all traffic types. These results could prove particularly interesting in packetbased multimedia networks, since packets in such networks have usually variable-length sizes and their service times have thus to be modeled as generally distributed variables. Furthermore, in many delay-sensitive real-time applications (e.g. transmission of voice over packet-based networks) loss of information is caused mainly due to packets arriving too late at the receiver, rather than buffer overflow since the buffers used in packet-based networks (e.g. IP networks) are usually quite large. Therefore, the modeling of the buffer space as infinite is acceptable.

The remainder of this paper is structured as follows. In the following section, we present the 
mathematical model. In section 3 , we analyze the system content at service initiation epochs. This is necessary for the analysis of the steady-state packet delay, which is further described in section 4. In section 5, we calculate the moments and tail probabilities of the packet delay of all priority classes, while we demonstrate our results by means of some numerical examples and touch upon an application in section 6. Finally, some conclusions are formulated in section 7 .

\section{Mathematical model}

We consider a discrete-time single-server queueing system with infinite buffer space. Time is assumed to be slotted. There are $M$ types of traffic arriving in the system. We denote the number of packet arrivals of class- $j$ during slot $k$ by $a_{j, k}(j=1, \ldots, M)$. The numbers of all types of packet arrivals are assumed to be i.i.d. from slot-to-slot and the numbers of per-slot arrivals are characterized by the (common) joint pgf

$$
A(\mathbf{z}) \triangleq \mathrm{E}\left[\prod_{j=1}^{M} z_{j}^{a_{j, k}}\right]
$$

with $\mathbf{z}$ defined as a vector with elements $z_{j}(j=1, \ldots, M)$. We define the marginal pgfs of the numbers of arrivals from class- $j$ during a slot by

$$
A_{j}(z) \triangleq \mathrm{E}\left[z^{a_{j, k}}\right]=\left.A(\mathbf{z})\right|_{z_{j}=z, z_{i}=1, i \neq j}
$$

We furthermore denote the arrival rate of class- $j$ packets $(j=1, \ldots, M)$ by $\lambda_{j}=A_{j}^{\prime}(1)$ and the total arrival rate by $\lambda_{T}=\sum_{j=1}^{M} \lambda_{j}$.

The service times of the class- $j$ packets are assumed to be i.i.d. and are characterized by the common probability mass function $s_{j}(m), m \geq 1$, and common pgf

$$
S_{j}(z) \triangleq \sum_{m=1}^{\infty} s_{j}(m) z^{m},
$$

with $j=1, \ldots, M$. We furthermore denote the mean service time of a class- $j$ packet by $\mu_{j}=$ 
$S_{j}^{\prime}(1)$ and define the load offered by class- $j$ packets as $\rho_{j} \triangleq \lambda_{j} \mu_{j}$. The total load is given by $\rho_{T} \triangleq \sum_{j=1}^{M} \rho_{j}$

The system has one server that provides for the transmission of the packets. Class- $i$ packets are assumed to have non-preemptive priority over class- $j$ packets when $i<j$, and within one class the service discipline is FCFS. The equilibrium condition requires that $\rho_{T}<1$.

\section{System content at service initiation epochs}

In order to be able to analyze the packet delay, we first analyze the system content at the beginning of so-called start-slots, i.e., slots at the beginning of which a packet (if available) can enter the server. Note that every slot during which the system is empty is also a start-slot. We denote the system content $n_{j, l}$ as the number of class- $j(j=1, \ldots, M)$ packets in the buffer at the beginning of the $l$-th start-slot, including the packet being served (if any). Their joint pgf is denoted by

$$
N_{l}(\mathbf{z}) \triangleq \mathrm{E}\left[\prod_{j=1}^{M} z_{j}^{n_{j, l}}\right]
$$

The set $\left\{\left(n_{1, l}, \ldots, n_{M, l}\right), l \geq 1\right\}$ forms a Markov chain, since the numbers of per-slot arrivals are i.i.d. and the buffer solely contains entire packets at the beginning of start-slots. $s_{l}^{*}$ denotes the service time of the packet that enters service at the beginning of start-slot $l$ (which corresponds - by definition - to regular slot $k$ ). We then establish the following system equations:

- If $n_{1, l}=\ldots=n_{M, l}=0$ :

$$
n_{i, l+1}=a_{i, k} \quad \text { for } i=1, \ldots, M \text {. }
$$

At the beginning of start-slot $l$, the system is empty in this case. The following slot is the next start-slot and the system content at the beginning of this slot consists of the new arrivals during slot $k$. 
- If $n_{1, l}=\ldots=n_{j-1, l}=0, n_{j, l}>0$ :

$$
n_{i, l+1}=n_{i, l}-1_{i=j}+\sum_{m=0}^{s_{l}^{*}-1} a_{i, k+m} \quad \text { for } i=1, \ldots, M,
$$

for $j=1, \ldots, M .1_{X}$ is the indicator function of $X$. A packet of the highest priority class present in the system at the beginning of start-slot $l$ (class- $j$ ) starts service at the beginning of this slot. The service ends $s_{l}^{*}$ slots later, at the beginning of start-slot $l+1$. The system content at the beginning of start-slot $l+1$ thus equals the superposition of the system content at the beginning of start-slot $l$ and the new arrivals during the class- $j$ service time minus the class- $j$ packet itself. The latter packet leaves the system just before start-slot $l+1$.

Using the system equations, we can derive a relation between $N_{l}(\mathbf{z})$ and $N_{l+1}(\mathbf{z})$. We assume that the system is stable (implying that the equilibrium condition $\rho_{T}<1$ is satisfied) and as a result $N_{l}(\mathbf{z})$ and $N_{l+1}(\mathbf{z})$ converge both to a common steady-state value $N(\mathbf{z})$ for $l \rightarrow \infty$. By taking the $l \rightarrow \infty$ limit of the relation between $N_{l}(\mathbf{z})$ and $N_{l+1}(\mathbf{z})$, we obtain:

$$
N(\mathbf{z})=\frac{z_{1}}{z_{1}-S_{1}(A(\mathbf{z}))}\left\{\frac{z_{M} A(\mathbf{z})-S_{M}(A(\mathbf{z}))}{z_{M}} N(\mathbf{0})+\sum_{j=2}^{M}\left[\frac{S_{j}(A(\mathbf{z}))}{z_{j}}-\frac{S_{j-1}(A(\mathbf{z}))}{z_{j-1}}\right] N\left(\mathbf{z}_{\mathbf{j}}\right)\right\},
$$

where $\mathbf{z}_{\mathbf{j}}$ denotes the $M$-dimensional vector $\left(0, \ldots, 0, z_{j}, \ldots, z_{M}\right)$ and $\mathbf{0}$ denotes a vector of size $M$ with all elements equal to 0 . There are $M$ quantities yet to be determined in the right hand side of equation (3), namely the functions $N\left(\mathbf{z}_{\mathbf{j}}\right)(j=2, . ., M)$ and the constant $N(\mathbf{0})$. First, we will recursively express $N\left(\mathbf{z}_{\mathbf{m}}\right)(m=1, . ., M)$ in terms of the $N\left(\mathbf{z}_{\mathbf{j}}\right)(j=m+1, . ., M)$ and $N(\mathbf{0})$. We define

$$
X_{0}(\mathbf{z}) \triangleq A(\mathbf{z})
$$


In the $m$-th step of our (recursive) calculation, we assume that $X_{m-1}\left(\mathbf{z}_{\mathbf{m}}\right)$ has already been defined and that the following equation holds:

$$
\begin{aligned}
N\left(\mathbf{z}_{\mathbf{m}}\right)= & \frac{z_{m}}{z_{m}-S_{m}\left(X_{m-1}\left(\mathbf{z}_{\mathbf{m}}\right)\right)}\left\{\frac{z_{M} X_{m-1}\left(\mathbf{z}_{\mathbf{m}}\right)-S_{M}\left(X_{m-1}\left(\mathbf{z}_{\mathbf{m}}\right)\right)}{z_{M}} N(\mathbf{0})\right. \\
& \left.+\sum_{j=m+1}^{M}\left[\frac{S_{j}\left(X_{m-1}\left(\mathbf{z}_{\mathbf{m}}\right)\right)}{z_{j}}-\frac{S_{j-1}\left(X_{m-1}\left(\mathbf{z}_{\mathbf{m}}\right)\right)}{z_{j-1}}\right] N\left(\mathbf{z}_{\mathbf{j}}\right)\right\} .
\end{aligned}
$$

Substituting $m=1$ in this equation yields equation (3), which is the starting point of our recursive procedure. Applying Rouché's theorem, it can then be proved that for given values of $z_{j}$ with $\left|z_{j}\right|<1(j=m+1, . ., M)$, the equation $z_{m}=S_{m}\left(X_{m-1}\left(\mathbf{z}_{\mathbf{m}}\right)\right)$ has a unique solution in the complex unit circle for $z_{m}$, which will be denoted by $Y_{m}\left(\mathbf{z}_{\mathbf{m}+\mathbf{1}}\right)$ in the remainder, and which is implicitly defined by

$$
\left.Y_{m}\left(\mathbf{z}_{\mathbf{m}+\mathbf{1}}\right) \triangleq S_{m}\left(X_{m-1}\left(\mathbf{z}_{\mathbf{m}}\right)\right)\right|_{z_{m}=Y_{m}\left(\mathbf{z}_{\mathbf{m}+\mathbf{1}}\right)} .
$$

Since any pgf is finite inside the unit circle and since $Y_{m}\left(\mathbf{z}_{\mathbf{m}+\mathbf{1}}\right)$ is a zero of the denominator of the right hand side of (4), $Y_{m}\left(\mathbf{z}_{\mathbf{m}+\mathbf{1}}\right)$ must also be a zero of the numerator. Defining

$$
\left.X_{m}\left(\mathbf{z}_{\mathbf{m}+\mathbf{1}}\right) \triangleq X_{m-1}\left(\mathbf{z}_{\mathbf{m}}\right)\right|_{z_{m}=Y_{m}\left(\mathbf{z}_{\mathbf{m}+\mathbf{1}}\right)}
$$

- and $X_{0}\left(\mathbf{z}_{1}\right)=A(\mathbf{z})$ - this leads to

$$
\begin{aligned}
N\left(\mathbf{z}_{\mathbf{m}+\mathbf{1}}\right)= & \frac{z_{m+1}}{z_{m+1}-S_{m+1}\left(X_{m}\left(\mathbf{z}_{\mathbf{m}+\mathbf{1}}\right)\right)}\left\{\frac{z_{M} X_{m}\left(\mathbf{z}_{\mathbf{m}+\mathbf{1}}\right)-S_{M}\left(X_{m}\left(\mathbf{z}_{\mathbf{m}+\mathbf{1}}\right)\right)}{z_{M}} N(\mathbf{0})\right. \\
& \left.+\sum_{j=m+2}^{M}\left[\frac{S_{j}\left(X_{m}\left(\mathbf{z}_{\mathbf{m}+\mathbf{1}}\right)\right)}{z_{j}}-\frac{S_{j-1}\left(X_{m}\left(\mathbf{z}_{\mathbf{m}+\mathbf{1}}\right)\right)}{z_{j-1}}\right] N\left(\mathbf{z}_{\mathbf{j}}\right)\right\}
\end{aligned}
$$

Note that this equals expression (4) with $m$ substituted by $m+1$, which means that the $m+1$-th step of the algorithm can be applied next. 
After $M-1$ iterations we finally find:

$$
N\left(\mathbf{z}_{\mathbf{M}}\right)=N(\mathbf{0}) \frac{z_{M} X_{M-1}\left(\mathbf{z}_{\mathbf{M}}\right)-S_{M}\left(X_{M-1}\left(\mathbf{z}_{\mathbf{M}}\right)\right)}{z_{M}-S_{M}\left(X_{M-1}\left(\mathbf{z}_{\mathbf{M}}\right)\right)} .
$$

Next, we can calculate the functions $N\left(\mathbf{z}_{\mathbf{m}}\right)(m=1, \ldots, M-1)$ as a function of $N(\mathbf{0})$. We therefore iteratively substitute the (in that step already) found expressions of $N\left(\mathbf{z}_{\mathbf{j}}\right)(j=$ $m+1, \ldots, N)$ in equation (4). Equaling $m$ to 1 finally gives $N(\mathbf{z})$ as a function of $N(\mathbf{0})$. The expression for general $M$ is too elaborate though (for general $\mathbf{z}$ ), but we have outlined the principle by which this $M$-th dimensional function can be calculated.

The last remaining unknown is $N(\mathbf{0})$. This constant can be calculated by applying the normalization condition $N(\mathbf{1})=1$, with $\mathbf{1}$ a vector of size $M$ with all elements equal to 1 . Substituting $\mathbf{z}$ by $\mathbf{1}$ in (3) and repeating the (iterative) calculation of $N(\mathbf{z})$ (in the special case $\mathbf{z}=\mathbf{1}$ ) gives (after some elaborate calculations)

$$
N(\mathbf{0})=\frac{1-\rho_{T}}{1-\rho_{T}+\lambda_{T}} .
$$

This concludes the procedure to calculate $N(\mathbf{z})$, which is used in the analysis of the packet delay in the next section.

\section{Packet Delay}

The delay of a packet is defined as the number of slots between the end of the packet's arrival slot and the end of its departure slot. We denote the delay of a tagged class- $j$ packet by $d_{j}$ and its pgf by $D_{j}(z)(j=1, \ldots, M)$. We furthermore denote the arrival slot of the tagged packet by slot $k$. If slot $k$ is a start-slot, it is assumed to be start-slot $l$. If slot $k$ is not a start-slot on the other hand, the last start-slot preceding slot $k$ is assumed to be start-slot $l$. In this section, we show how an expression for $D_{j}(z)$ - for general $j$ - is derived.

Let us first refer to the packets in the system at the end of slot $k$, but that have to be served 
before the tagged packet as the "primary packets". So, basically, the tagged class- $j$ packet enters the server, when all primary packets and all packets with higher priority that arrived after slot $k$ (i.e., while the tagged packet is waiting in the queue) are transmitted. In order to analyze the delay of the tagged class- $j$ packet, the number of packets that are served between the arrival slot of the tagged class- $j$ packet and its departure slot is important (and more specifically the time necessary to transmit them), not the precise order in which they are served. Therefore, in order to facilitate the analysis, we consider an equivalent virtual system with an altered service discipline. From slot $k$ on, we aggregate the $j-1$ highest priority classes in one class and serve the packets in this aggregated class in a LCFS way (those in the queue at the end of slot $k$ and newly arriving ones). So, a primary packet can enter the server, when the system becomes free (for the first time) of packets of this aggregated class that arrived during and after the service time of the primary packet that preceded it according to the new service discipline. Let $v_{i, m}^{(n)}$ $(i=1, \ldots, j)$ denote the length of the time period during which the server is occupied by the $m$-th class- $i$ packet that arrives during slot $n$ and its "successors" of the aggregated class, i.e., the time period starting at the beginning of the service of that packet and terminating when the system becomes free (for the first time) of packets of the $j-1$ highest priority classes which arrived during and after its service time. The $v_{i, m}^{(n)}$ s $(i=1, \ldots, j)$ are called sub-busy periods, initiated by the $m$-th class- $i$ packet that arrived during slot $n$. Note that the $v_{i, m}^{(n)}$ depend on the class we are analyzing (i.e. class- $j$ ), but to alleviate the notation this dependency is taken into account only implicitly. It is clear that the lengths of consecutive sub-busy periods initiated by class $i$ packets are i.i.d. and thus have the same pgf $V_{i}(z)$ (which implicitly depends on $j$ ). This pgf is given by

$$
V_{i}(z)=S_{i}\left(z A\left(V_{1}(z), \ldots, V_{j-1}(z), 1, \ldots, 1\right)\right)
$$

with $i=1, \ldots, j ; j=1, \ldots, M$. This can be understood as follows: when the $m$-th class- $i$ packet that arrived during slot $n$ enters service, its sub-busy period, $v_{i, m}^{(n)}$, consists of two parts: 
the service time of that packet itself, and the service times of the packets of higher priority than the tagged class- $j$ packet (the aggregated class) that arrive during its service time and of their successors of the aggregated class. This leads to equation (7).

Finally, $d_{j}$ can be expressed in terms of the $n_{i, l}, i=1, \ldots, M$ defined in the previous section. $D_{j}(z)$ is then calculated as a function of $N(\mathbf{z})$ by $z$-transforming this expression for $d_{j}$. The function $N(\mathbf{z})$ was already calculated in section 3 and as a result $D_{j}(z)$ can be found. We refer to Walraevens et al. (2002) and Bruneel and Kim (1993) for more details on similar queueing analyses. $D_{j}(z)$ is found to be given by (after some extensive mathematical manipulations)

$$
\begin{aligned}
D_{j}(z)= & \frac{1-\sum_{i=1}^{j} \rho_{i}}{\lambda_{j}} \frac{S_{j}(z)\left(z B_{j-1}(z)-1\right)}{z B_{j-1}(z)-B_{j}(z)} \frac{B_{j}(z)-B_{j-1}(z)}{V_{j}(z)-1} \\
& \times\left(1-\frac{\sum_{i=j+1}^{M} \rho_{i}}{1-\sum_{i=1}^{j} \rho_{i}}+\frac{1}{1-\sum_{i=1}^{j} \rho_{i}} \sum_{i=j+1}^{M} \rho_{i} \frac{V_{i}(z)-1}{\mu_{i}\left(z B_{j-1}(z)-1\right)}\right),
\end{aligned}
$$

with expression (7) of the $V_{i}(z)$ extended to $i=j+1, \ldots, M$ and with

$$
B_{i}(z) \triangleq A\left(V_{1}(z), \ldots, V_{i}(z), 1, \ldots, 1\right)
$$

$i=0, \ldots, M$.

Note that expression (8) is also correct for $D_{1}(z)$, the pgf of the highest priority class.

\section{$5 \quad$ Performance measures}

In this section, we will show how important performance measures such as moments and tail probabilities of the class- $j$ delay (for all $j$ ) can be calculated from the obtained pgf $(8)$ of $D_{j}(z)$.

\section{$5.1 \quad$ Moments}

All moments of the packet delays can be calculated by taking the necessary derivatives of expression (8), $j=1, \ldots, M$ and substituting $z$ by 1 , due to the moment generating property of pgfs. The functions $V_{i}(z)$, defined in the previous section, can be explicitly found only in case of 
some specific arrival and service processes. However, since the $V_{i}(z)$ are pgfs, $V_{i}(1)=1$ and as a result the derivatives of the $V_{i}(z)$ for $z=1$, necessary to calculate the moments of the packet delays can be calculated in closed-form.

Let us define $\lambda_{i j}$ and $\mu_{j j}$ as

$$
\left.\lambda_{i j} \triangleq \frac{\partial^{2} A\left(z_{1}, \ldots, z_{M}\right)}{\partial z_{i} \partial z_{j}}\right|_{z_{1}=\ldots=z_{M}=1}
$$

and

$$
\left.\mu_{j j} \triangleq \frac{d^{2} S_{j}(z)}{d z^{2}}\right|_{z=1}
$$

respectively, with $i, j=1, \ldots, M$. Now we can calculate the mean packet delay of class- $j$ by taking the first derivative of $D_{j}(z)$ for $z=1$. We find

$$
\begin{aligned}
E\left[d_{j}\right]= & \mu_{j}+\frac{\lambda_{j j} \mu_{j}}{2 \lambda_{j}\left(1-\sum_{m=1}^{j} \rho_{m}\right)}+\frac{\sum_{m=1}^{j} \lambda_{m j} \mu_{m}}{\lambda_{j}\left(1-\sum_{m=1}^{j} \rho_{m}\right)}+\frac{\sum_{m, n=1}^{j-1} \lambda_{m n} \mu_{m} \mu_{n}}{2\left(1-\sum_{m=1}^{j-1} \rho_{m}\right)\left(1-\sum_{m=1}^{j} \rho_{m}\right)} \\
& +\frac{\sum_{m=1}^{M} \lambda_{m} \mu_{m m}}{2\left(1-\sum_{m=1}^{j-1} \rho_{m}\right)\left(1-\sum_{m=1}^{j} \rho_{m}\right)} .
\end{aligned}
$$

In a similar way, expressions for the variance (and higher order moments) of the packet delays of all classes can be established by taking the appropriate derivatives of the respective pgfs as well. These expressions are too elaborate to include in this text, but in order to show that their calculation is feasible and has been implemented, we will show a plot of the variance of the packet delay in section 6 .

\subsection{Tail probabilities}

Furthermore, the probability mass functions of the packet delays can also be approximately calculated from the pgf's calculated in the previous section. More precisely, $\operatorname{Prob}\left[d_{j}=n\right]$ can 
be approximated by the dominant singularity approach and this approximation is better for higher $n$. These so-called tail distributions $\left(\operatorname{Prob}\left[d_{j}=n\right]\right.$ for high $\left.n\right)$ are often used to impose statistical bounds on the guaranteed QoS for both classes.

In order to determine the asymptotic behavior of the tail distribution, the dominant singularity of the respective pgf is important. Analyzing the tail behavior of the delay of class- $j$ packets is a bit more involved than in usual queueing analyses, since it is not a priori clear what the dominant singularity of $D_{j}(z)$ is. This is due to the occurrence of the functions $V_{i}(z)$, $i=1, \ldots, j-1$ in (8), which are only implicitly defined (see expression (7)). Laevens and Bruneel (1998) have proved that these implicitly defined functions have a branch-point singularity $z_{B}$ where their first derivatives become infinite but the functions themselves remain finite. In the neighbourhood of $z_{B}, V_{i}(z)$ can approximately be written as

$$
V_{i}(z) \approx V_{i}\left(z_{B}\right)-K_{V_{i}}\left(z_{B}-z\right)^{1 / 2}
$$

with

$$
K_{V_{i}}=\sqrt{\frac{2 B_{j-1}\left(z_{B}\right)\left(S_{i}^{\prime}\left(z_{B} B_{j-1}\left(z_{B}\right)\right)\right)^{2} / z_{B}}{\sum_{m=1}^{j-1} B_{j-1}^{(m)}\left(z_{B}\right) S_{m}^{\prime \prime}\left(z_{B} B_{j-1}\left(z_{B}\right)\right)+\sum_{m=1}^{j-1} \sum_{n=1}^{j-1} B_{j-1}^{(m n)}\left(z_{B}\right) S_{m}^{\prime}\left(z_{B} B_{j-1}\left(z_{B}\right)\right) S_{n}^{\prime}\left(z_{B} B_{j-1}\left(z_{B}\right)\right)}} .
$$

Here $B_{i}(z)$ is defined as before,

$$
\left.B_{i}^{(m)}(z) \triangleq \frac{\partial A\left(z_{1}, \ldots, z_{M}\right)}{\partial z_{m}}\right|_{z_{1}=V_{1}(z), \ldots, z_{i}=V_{i}(z), z_{i+1}=\ldots=z_{M}=1}
$$

and

$$
\left.B_{i}^{(m n)}(z) \triangleq \frac{\partial^{2} A\left(z_{1}, \ldots, z_{M}\right)}{\partial z_{m} \partial z_{n}}\right|_{z_{1}=V_{1}(z), \ldots, z_{i}=V_{i}(z), z_{i+1}=\ldots=z_{M}=1}
$$

$z_{B}$ is then also a branch point of $D_{j}(z)$. A second potential singularity of $D_{j}(z)$ is given by 
the (dominant) zero $z_{P}$ of $z B_{j-1}(z)-B_{j}(z)$ on the real axis (see expression (8)).

The tail behavior of the packet delay of class- $j$ packets is thus characterized by the singularities $z_{P}$ or $z_{B}$, depending on which one is dominant (i.e., which one has the smallest modulus). Three types of tail behavior may occur, namely one type when $z_{P}$ is dominant, a second type when $z_{B}$ is dominant and yet another type when $z_{P}=z_{B}$. In those three cases, the tail probabilities of the class- $j$ packet delay are given by (see Laevens and Bruneel (1998) for more details)

$$
\operatorname{Prob}\left[d_{j}=n\right] \approx \begin{cases}\frac{K_{1} z_{P}^{-n+1}}{\frac{K_{2} n^{-1 / 2} z_{B}^{-n}}{\sqrt{z_{B} \pi}}} & \text { if } z_{P} \text { dominant } \\ \frac{K_{3} n^{-3 / 2} z_{B}^{-n}}{2 \sqrt{\pi / z_{B}}} & \text { if } z_{B} \text { dominant. }\end{cases}
$$

The constants $K_{i}(i=1,2,3)$ can be found by investigating the pgf $D_{j}(z)$ in the neighborhood of its singularity. They are given by

$$
\begin{aligned}
K_{1}= & \frac{S_{j}\left(z_{P}\right) B_{j-1}\left(z_{P}\right)\left(z_{P}-1\right)\left(\left(1-\rho_{T}\right)\left(z_{P} B_{j-1}\left(z_{P}\right)-1\right)+\sum_{i=j+1}^{M} \lambda_{i}\left(V_{i}\left(z_{P}\right)-1\right)\right)}{\lambda_{j}\left(V_{j}\left(z_{P}\right)-1\right)\left(\sum_{m=1}^{j} B_{j}^{(m)}\left(z_{P}\right) V_{m}^{\prime}\left(z_{P}\right)-B_{j-1}\left(z_{P}\right)-z_{P} \sum_{m=1}^{j-1} B_{j-1}^{(m)}\left(z_{P}\right) V_{m}^{\prime}\left(z_{P}\right)\right)} \\
K_{2}= & \frac{S_{j}\left(z_{B}\right) B_{j-1}\left(z_{B}\right)\left(z_{B}-1\right)\left(\left(1-\rho_{T}\right)\left(z_{B} B_{j-1}\left(z_{B}\right)-1\right)+\sum_{i=j+1}^{M} \lambda_{i}\left(V_{i}\left(z_{B}\right)-1\right)\right)}{\lambda_{j}\left(V_{j}\left(z_{B}\right)-1\right)\left(\sum_{m=1}^{j} K_{V_{m}} B_{j}^{(m)}\left(z_{B}\right)-z_{B} \sum_{m=1}^{j-1} K_{V_{m}} B_{j-1}^{(m)}\left(z_{B}\right)\right)} \\
K_{3}= & \frac{S_{j}\left(z_{B}\right)}{\lambda_{j}\left(z_{B} B_{j-1}\left(z_{B}\right)-B_{j}\left(z_{B}\right)\right)^{2}\left(V_{j}\left(z_{B}\right)-1\right)^{2}}\left[\left(z_{B}-1\right)\right. \\
& \times\left(\sum_{m=1}^{j-1} K_{V_{m}}\left[B_{j-1}\left(z_{B}\right)\left\{B_{j}^{(m)}\left(z_{B}\right)+B_{j}^{(j)}\left(z_{B}\right) S_{j}^{\prime}\left(z_{B} B_{j-1}\left(z_{B}\right)\right)\right\}-B_{j}\left(z_{B}\right) B_{j-1}^{(m)}\left(z_{B}\right)\right]\right) \\
& \times\left(\left(1-\rho_{T}\right)\left(z_{B} B_{j-1}\left(z_{B}\right)-1\right)+\sum_{i=j+1}^{M} \lambda_{i}\left(V_{i}\left(z_{B}\right)-1\right)\right)\left(V_{j}\left(z_{B}\right)-1\right) \\
& +\left(\sum_{m=1}^{j-1} K_{V_{m}} B_{j-1}^{(m)}\left(z_{B}\right)\right)\left(B_{j}\left(z_{B}\right)-B_{j-1}\left(z_{B}\right)\right)\left(z_{B} B_{j-1}\left(z_{B}\right)-B_{j}\left(z_{B}\right)\right) \\
& \times\left(\left\{\left(1-\rho_{T}\right) z_{B}+\sum_{i=j+1}^{M} \lambda_{i} S_{i}^{\prime}\left(z_{B} B_{j-1}\left(z_{B}\right)\right)\right\}\left\{V_{j}\left(z_{B}\right)-1\right\}\right.
\end{aligned}
$$




$$
\left.\left.-\left\{\left(1-\rho_{T}\right)\left(z_{B} B_{j-1}\left(z_{B}\right)-1\right)+\sum_{i=j+1}^{M} \lambda_{i}\left(V_{i}\left(z_{B}\right)-1\right)\right\} S_{j}^{\prime}\left(z_{B} B_{j-1}\left(z_{B}\right)\right)\right)\right] .
$$

The first expression of (12) shows a geometric tail behavior, while the second and third expression show non-geometric tail behaviors. Note that the pgf of the class-1 packet delay does not have a branch point (since $V_{1}(z)=S_{1}(z)$ is explicitly given) and thus the class-1 packet delay tail behavior is always dominated by the regular pole $z_{P}$.

\section{Numerical examples}

In this section, we present some numerical examples. Firstly, we show some straight-forward results for an output queueing packet switch. Secondly, we apply our results to a priority queue handling multimedia traffic.

\subsection{Output queueing packet switch}

We assume an output queueing switch with $N$ inlets and $N$ outlets as depicted in Figure 1. The packet arrivals on each inlet are assumed to be i.i.d., and generated by a Bernoulli process with arrival rate $\lambda_{T}$. An arriving packet is assumed to be of class- $j$ with probability $\lambda_{j} / \lambda_{T}$ $(j=1, \ldots, M)\left(\sum_{j=1}^{M} \lambda_{j}=\lambda_{T}\right)$. The incoming packets are then routed to the output queue corresponding to their destination, in an independent and uniform way. Therefore, the output queues behave identically and we can concentrate on the analysis of 1 output queue. In view of the previous, the arrivals of the $M$ types of packets to an output queue are generated according to an $M$-dimensional binomial process. It is fully characterized by the following joint pgf

$$
A\left(z_{1}, \ldots, z_{M}\right)=\left(1-\sum_{j=1}^{M} \frac{\lambda_{j}}{N}\left(1-z_{j}\right)\right)^{N}
$$

In the remainder of this section, we assume that $N=16$. We furthermore assume deterministic service times for class- $j$ equal to $\mu_{j}$ number of slots. Define $\alpha_{j}(j=1, \ldots, M)$ as the fraction of load of class- $j$ in the total traffic mix, i.e., $\alpha_{j}=\rho_{j} / \rho_{T}$. We will consider the number 


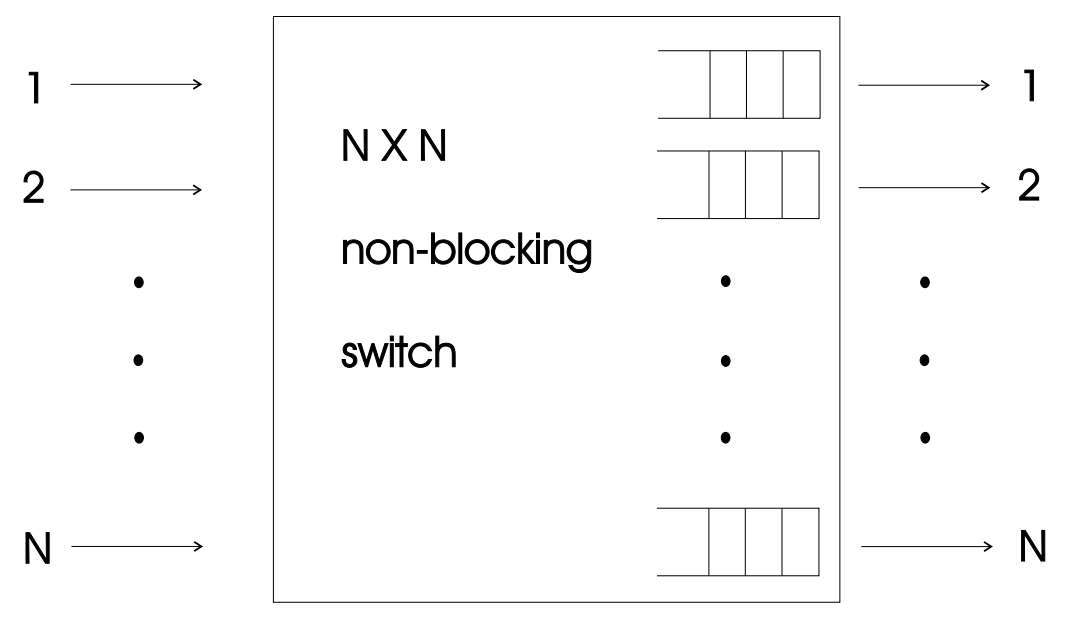

Figure 1: The output queueing packet switch

of classes $M$ in the following figures equal to 3 (but similar figures can be straight-forwardly plotted for a higher number of priority classes). We focus on the delay characteristics, because these are the most important characteristics in priority queues (from a practical point of view).

In Figure 2, the means and variances of the packet delays of class- $j$ packets $(j=1,2,3)$ are shown as functions of the total load $\rho_{T}$, when $\mu_{j}=2$ and for three combinations of the $\alpha_{j}$ 's. In order to compare with a FIFO scheduling, we have also shown the mean value and variance of the packet delay in that case. Since, in this example, the service times of all packets are equally distributed, the packet delay is then of course the same for all packets, and can thus be calculated as if there were only one class of packets arriving according to an arrival process with $\operatorname{pgf} A(z, z, z)$. This has already been analyzed, e.g., in Bruneel and Kim (1993). One can observe the influence of assigning priorities to the three classes: mean value and variance of the delay of high-priority packets reduces significantly. The price to pay is of course a larger mean value and variance of the delay for class- 2 and class- 3 packets. The mean delay of the lowest priority class is always higher than the mean delay when a FIFO scheduling is applied, while for class-2 packets it depends on the $\alpha_{j}$ 's and the total load whether their mean delay is larger or smaller than in the FIFO case. These figures also show that the mean and variance of the delay of all classes increases as the share of the high-priority traffic in the overall load increases.

Figure 3 shows the mean packet delays of the three classes as functions of the service time 


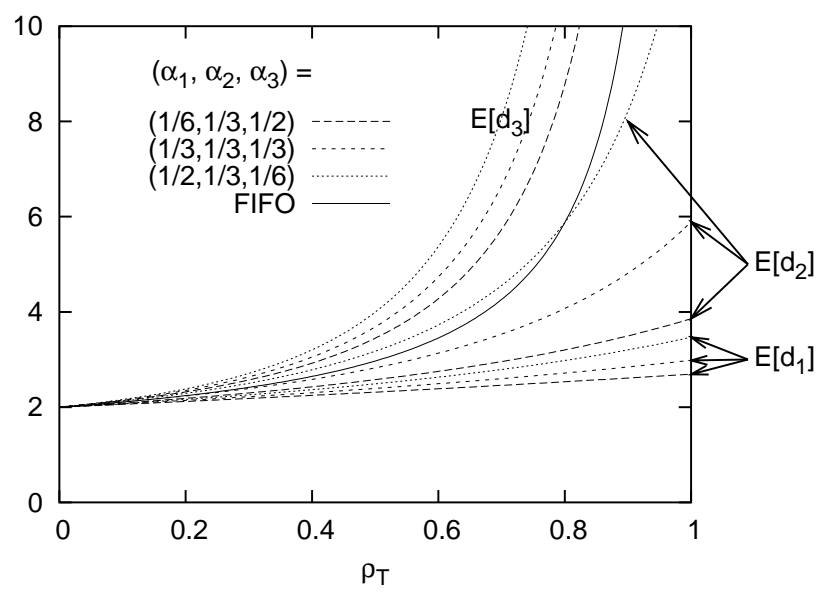

a. mean

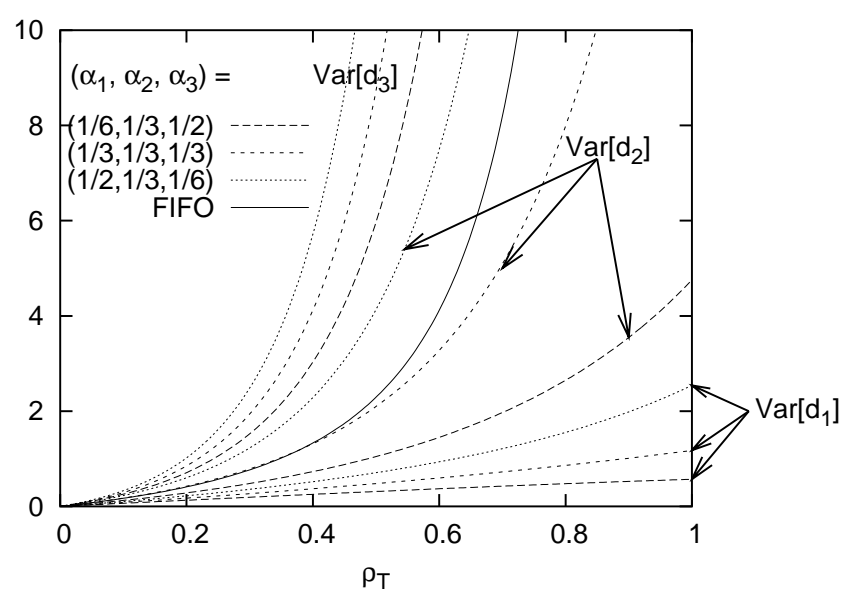

b. variance

Figure 2: Mean and variance of the packet delay versus the total load

of class- $j$ packets $(j=1,2,3)$ (Figures 3a., b. and c. respectively), when $\rho_{T}=0.75$, and the service times of the other packets are deterministically equal to 2 . We see that the mean packet delays of the three classes are proportional with $\mu_{1}$ (Figure 3a.) and that the impact of $\mu_{1}$ on the mean delays of the three priority classes is significant. Varying the mean service time of class-2 packets (see Figure 3b.) has a considerable influence on the mean delays of the class-2 and class-3 packets, while the influence on the mean packet delay of high priority packets is small, but not zero. The mean service time of the class-3 packets has only a minor impact on the mean delay of class- 1 and class- 2 packets, but not negligible, as can be deduced from Figure 3c. The behavior in the plots of Figure 3 can be explained as follows: changing the service process of a class has a big influence on the performance characteristics of this class and all classes with lower priority, due to the priority scheduling discipline. The influence on the performance characteristics of packets of higher priority is small, but not zero. Indeed, because the priority scheduling is non-preemptive, arriving higher priority packets cannot interrupt a packet in service, even if it is a packet of lower priority. The longer the (mean) service times of these lower priority packets, the bigger the (mean) packet delay of the higher priority packets.

In the next figures, we are going to examine the tail behavior of the packet delay. We have shown in subsection 5.2 , that the tail probabilities of the class- $j$ delay $(j \geq 2)$ can have 3 types of behavior, depending on which singularity of $D_{j}(z)$ is dominant. In this paragraph we will 


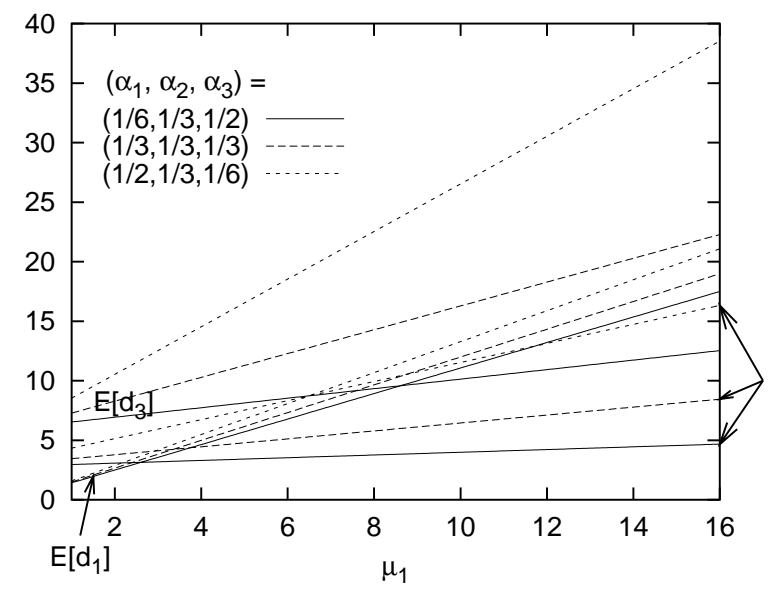

a. versus $\mu_{1}$

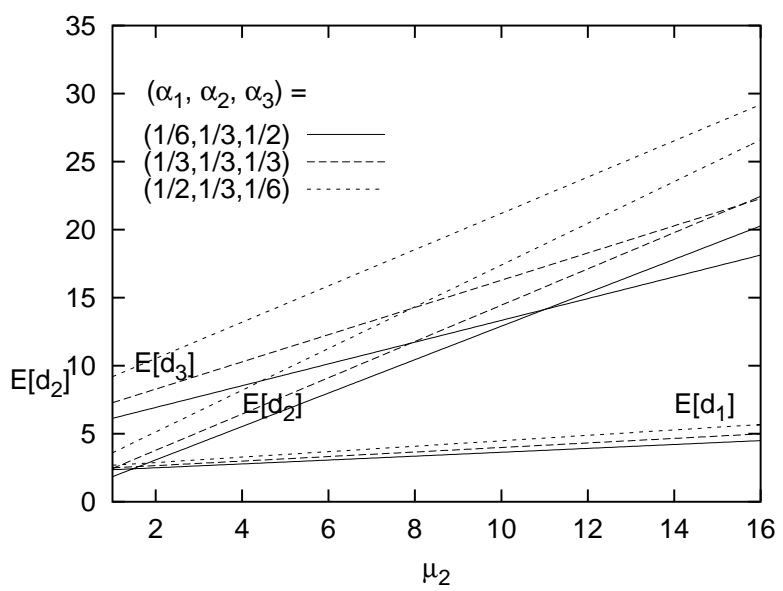

b. versus $\mu_{2}$

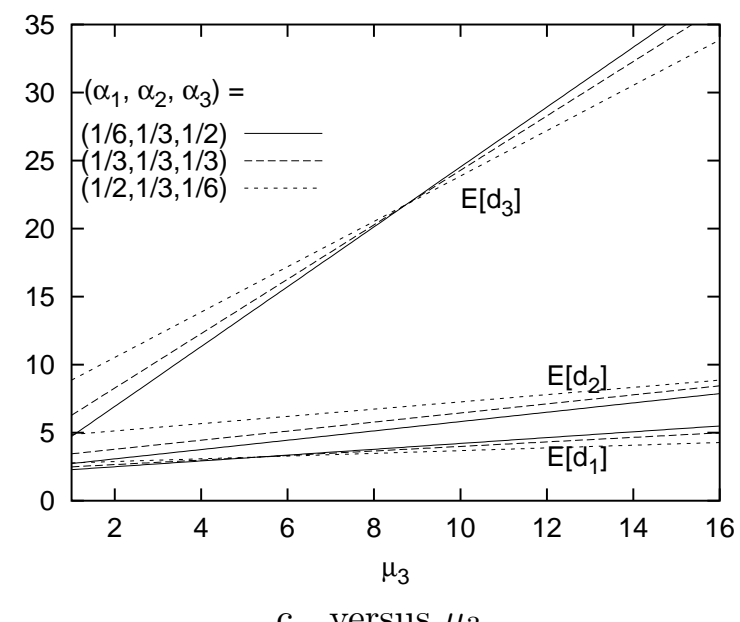

c. versus $\mu_{3}$

Figure 3: Mean packet delay versus the mean service time of class-1, class-2 or class-3 packets 


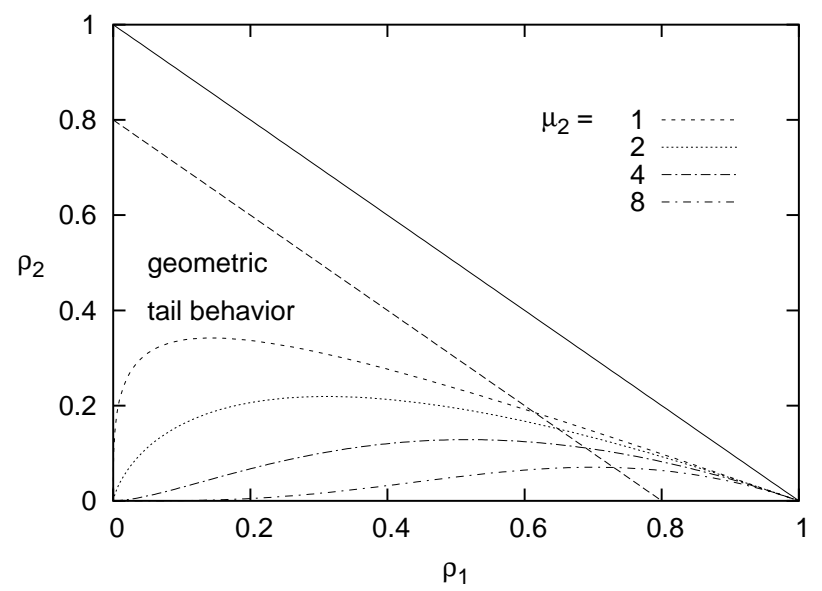

Figure 4: Regions for tail behavior as a function of the load of class-1 and class-2

discuss the 3 types of behavior specifically for the class- 2 packet delay. Of course, the same can be done for the class-3 packet delay (or in case of a general number of priority classes, for all priority classes). In case of the arrival process considered in this section (with 3 priority classes), Figure 4 shows for which combinations of class- 1 and class-2 loads the transition type behavior occurs for the class-2 packet delay when $\mu_{1}=2$ and for various values of $\mu_{2}$, i.e., for which combination of loads the regular pole and the branch point coincide. The service times of class- 1 and class-2 packets are deterministic. Note that the parameters of the lower priority classes do not play a role in this, except for imposing an upper bound on $\rho_{1}+\rho_{2}$. In the area above the upper line defined by $\rho_{1}+\rho_{2}=1$ in Figure 4 , the total load of class- 1 and class- 2 is larger than 1, and as a result, the system is definitely unstable above that line (even when no class-3 packets arrive). For positive $\rho_{3}$ the stability borderline lowers. The lower line for instance gives an upper bound of combinations of $\rho_{1}$ and $\rho_{2}$ when the load of class- 3 is 0.2 . In the region above the curves, the tail behavior is geometric for the respective $\rho_{1}$ and $\rho_{2}$, while below the curves the tail behavior is typically non-geometric. As can be seen, the higher the mean service time of class- 2 packets, the smaller the region where the tail behavior is non-geometric.

Figure 5 shows the tail behavior of the packet delay for deterministic service times $\left(\mu_{1}=\mu_{2}=\right.$ $\mu_{3}=2$ ). Figure 5a. shows the tail probabilities of the class-2 packet delay for $\rho_{1}=\rho_{3}=0.2$ and $\rho_{2}=0.02$ (non-geometric behavior), approximately 0.2 (transition) and 0.4 (geometric 


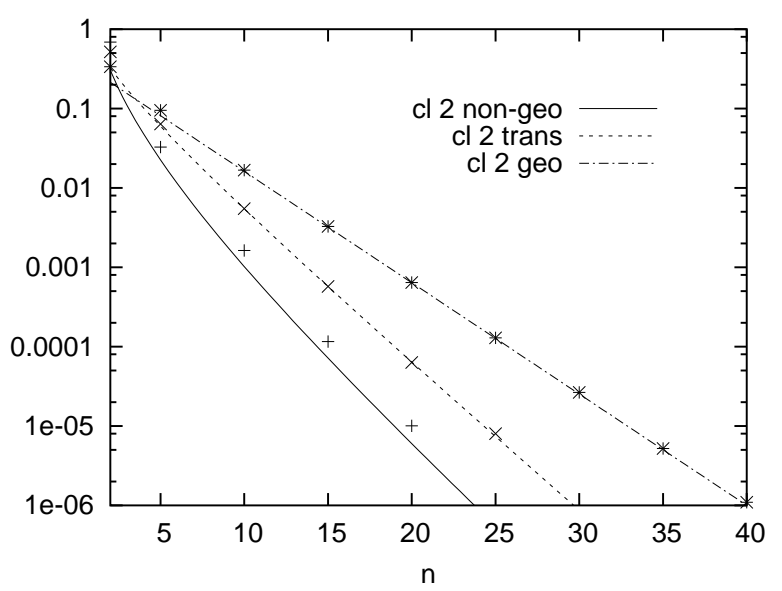

a. Class-2 packet delay

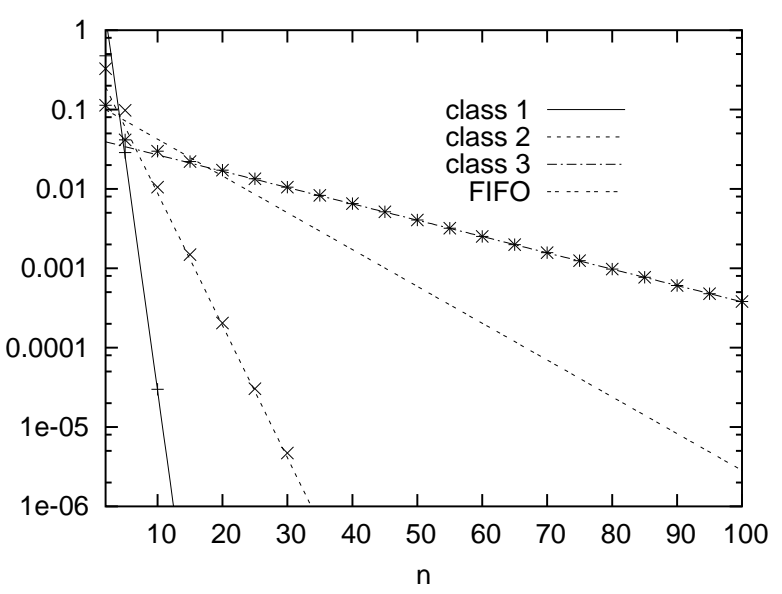

b. Packet delay of all classes

Figure 5: Tail probabilities of the packet delay

behavior) respectively. One can see that the curves for the former two cases are not straight lines, thus showing their non-geometric property. We have compared our approximations with simulation results (marks in the figures). The figure shows that the approximations for the tail probabilities are very good. Figure 5b. depicts the tail behavior of the delay of the three classes for deterministic service times if $\rho_{1}=0.2, \rho_{2}=0.3$ and $\rho_{3}=0.4$. We have also shown the tail probabilities of the packet delay when the scheduling discipline is FIFO instead of a priority scheduling discipline (for the same arrival and service time characteristics). This shows the impact of giving an amount of traffic higher priority on the delay of the different priority classes: the delay of high-priority packets is highly reduced, while the delay of the low-priority packets is larger than for the FIFO case. In this case, the delay of class-2 packets is also reduced.

\subsection{A priority queue handling multimedia traffic}

In this subsection, we assume traffic of three traffic classes being handled by a queue, namely a class consisting of voice traffic, one of Video-on-Demand (VoD) traffic and a third one of data traffic. We call these class-1, class-2 and class-3 respectively in the remainder. Evidently, an interactive voice application will have the most stringent delay requirements while data (file transfer) will have the loosest delay bounds, with VoD somewhere in between. This is reflected by the priority level that has been assigned to each of the three traffic classes. The numbers of per-slot arrivals of class- $j$ are distributed according to a Poisson process with arrival rate $\lambda_{j}$. 
We furthermore assume deterministic service times for class- $j$ equal to $\mu_{j}$ number of slots. The arriving packets are transmitted via an output link. We assume that this link has a transmission rate of $620 \mathrm{Mb} / \mathrm{s}$. The video and data packets all carry 1500 bytes corresponding to the length of an Ethernet packet. Due to the relatively low bitrate of a voice codec $(8-64 \mathrm{~kb} / \mathrm{s})$, the filling time of a voice packet can become significant if the packet length is too high; as a result voice packets are usually kept small and are chosen to be equal to 200 bytes in this section. The slot-length $\Delta$ then equals the amount of time to transmit 100 bytes, or

$$
\Delta=\frac{8 \times 100}{620.10^{6}} \text { seconds }=1.29 \mu s
$$

Define $\alpha_{j}(j=1,2,3)$ again as the fraction of load of class- $j$ in the total traffic mix, i.e., $\alpha_{j}=\rho_{j} / \rho_{T}$.

In Figure 6a., the means of the packet delay of the class-1, class- 2 and class- 3 packets are shown as functions of the total load $\rho_{T}$ for $\alpha_{1}=0.05,0.1$ and 0.2 respectively and for $\alpha_{2}=0.4$. One can observe the influence of assigning priorities: mean delay of voice packets is kept as low as possible. Since the voice packets constitute a limited fraction of the traffic stream, the mean delay of the video packets is also kept relatively low. However, one can see that, especially for $\alpha_{1}=0.2$ and for high loads, the influence of the voice packets on the mean delay of the video packets is not negligible. The price to pay for limiting the (mean) delay of voice and video packets is a larger mean delay of the data packets (as expected). This figure also shows that the share of the high-priority traffic in the overall load can have a significant impact on the mean delays of all priority classes.

Figure 6b. shows the tail probabilities of the packet delay of the class-2 (video) packets with $\rho_{1}=0.05,0.1$ and 0.2 respectively and $\rho_{2}=0.4$. The value of $d_{2}$ is expressed in $\mu \mathrm{s}$ in this figure. For each value of $\rho_{1}$ we have plotted two curves. The lower curves depict the tail probabilities when no data traffic arrives (i.e, $\rho_{3}=0$ ). The upper curves show the tail probabilities when the bandwidth that is not used by the voice or video traffic is consumed entirely by data packets 


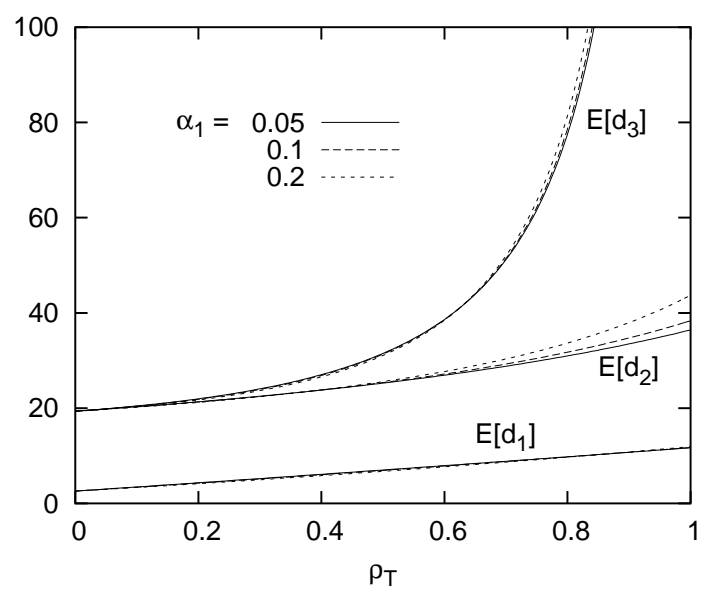

a. Mean packet delay (in $\mu$ s)

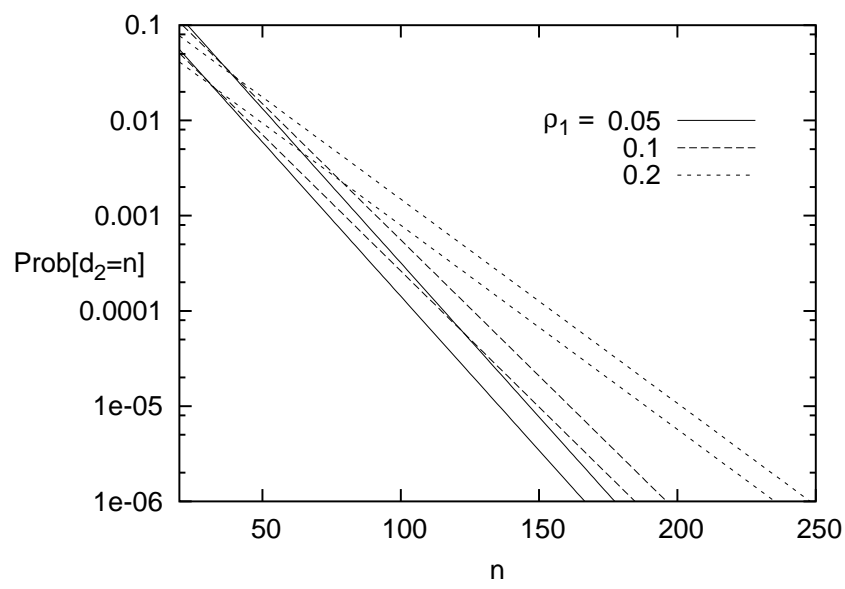

b. Tail probabilities of the class-2 delay (in $\mu \mathrm{s}$ )

Figure 6: Multimedia priority queue

$\left(\rho_{3}=1-\rho_{1}-\rho_{2}\right)$. We see that data packets have a non-negligible influence on the delay characteristics of video traffic (since the priority scheduling is non-preemptive and thus video packets that arrive during the transmission of a data packet have to wait until that packet is fully transmitted), although this influence remains limited (note that e.g. the slope of both curves are equal). The impact of the voice packets (or of load $\rho_{1}$ ) on the delay characteristics of the video packets is much larger. This was to be expected because of the priority given to voice packets over video packets.

\section{Conclusions}

In this paper, we analyzed the packet delays of all classes in a queueing system with a nonpreemptive (HOL) priority scheduling discipline and with a general number of priority classes. A generating-functions-approach was adopted, which led to closed-form expressions of the moments and accurate approximations of the probabilities of the packet delays of all the classes, that are easy to evaluate. The service times are class-based and generally distributed. Therefore, the results could be used to evaluate the system performance in packet-based networks, that support multiple applications to which different priorities are assigned. An example is touched upon wherein voice, video and data streams are multiplexed. 


\section{References}

Bruneel, H. and Kim, B. (1993). Discrete-time models for communication systems including ATM. Kluwer Academic Publisher, Boston.

Khamisy, A. and Sidi, M. (1992). Discrete-time priority queues with two-state Markov Modulated arrivals. Stochastic Models, 8(2):337-357.

Kleinrock, L. (1976). Queueing systems volume II: Computer applications. John Wiley \& Sons.

Laevens, K. and Bruneel, H. (1998). Discrete-time multiserver queues with priorities. Performance Evaluation, 33(4):249-275.

Liebeherr, J. and Wrege, D. (1999). Priority queue schedulers with approximate sorting in output-buffered switches. IEEE Journal on Selected Areas in Communications, 17(6):11271144.

Liu, K., Petr, D., Frost, V., Zhu, H., Braun, C., and Edwards, W. (1997). Design and analysis of a bandwidth management framework for ATM-based broadband ISDN. IEEE Communications Magazine, 35(5):138-145.

Miller, R. (1960). Priority queues. Annals of Mathematical Statistics, 31:86-103.

Parekh, A. and Gallager, R. (1994). A generalized processor sharing approach to flow-control in integrated services networks: the multiple node case. IEEE/ACM Transactions on Networking, 2(2):137-150.

Rubin, I. and Tsai, Z. (1989). Message delay analysis of multiclass priority TDMA, FDMA, and discrete-time queueing systems. IEEE Transactions on Information Theory, 35(3):637-647.

Stanford, D. (1991). Interdeparture-time distributions in the nonpreemptive priority $\sum \mathrm{M}_{\mathrm{i}} / \mathrm{G}_{\mathrm{i}} / 1$ queue. Performance Evaluation, 12(1):43-60.

Sugahara, A., Takine, T., Takahashi, Y., and Hasegawa, T. (1995). Analysis of a nonpreemptive priority queue with SPP arrivals of high-class. Performance Evaluation, 21(3):215-238. 
Takagi, H. (1991). Queueing analysis: a foundation of performance evaluation volume 1: vacation and priority systems, part 1. North-Holland.

Takahashi, Y. and Hashida, O. (1991). Delay analysis of discrete-time priority queue with structured inputs. Queueing Systems, 8(2):149-164.

Takine, T. (1996). A nonpreemptive priority MAP/G/1 queue with two classes of customers. Journal of Operations Research Society of Japan, 39(2):266-290.

Takine, T., Matsumoto, Y., Suda, T., and Hasegawa, T. (1994a). Mean waiting-times in nonpreemptive priority-queues with Markovian arrival and iid service processes. Performance Evaluation, 20(1-3):131-149.

Takine, T., Sengupta, B., and Hasegawa, T. (1994b). An analysis of a discrete-time queue for broad-band ISDN with priorities among traffic classes. IEEE Transactions on Communications, 42(2-4):1837-1845.

Tham, C., Yao, Q., and Jiang, Y. (2002). Achieving differentiated services through multi-class probabilistic priority scheduling. Computer Networks, 40(4):577-593.

Walraevens, J., Steyaert, B., and Bruneel, H. (2000). Performance analysis of the system contents in a discrete-time non-preemptive priority queue with general service times. Belgian Journal of Operations Research, Statistics and Computer Science (JORBEL), 40(1-2):91-103.

Walraevens, J., Steyaert, B., and Bruneel, H. (2002). Delay characteristics in discrete-time GI-G-1 queues with non-preemptive priority queueing discipline. Performance Evaluation, $50(1): 53-75$.

Walraevens, J., Steyaert, B., and Bruneel, H. (2003). Performance analysis of a single-server ATM queue with a priority scheduling. Computers $\& 3$ Operations Research, 30(12):1807-1829.

Xiao, X. and Ni, L. (1999). Internet QoS: a big picture. IEEE Network, 13(2):8-18. 